Rheumaplus 2013 · 12:2

DOI 10.1007/s12688-013-0056-6

(C) Springer-Verlag Wien 2013

\author{
B. Leeb \\ Springer-Verlag, Wien
}

\title{
Und wieder eine Premiere!
}

rheuma plus hat sein Erscheinungsbild mit der letzten Ausgabe geändert. Ich hoffe, das modernere Layout gefällt Ihnen genauso gut wie mir. Was die inhaltlichen Belange betrifft, so werden wir, Attila Dunky und ich, unsere Linie mit vor allem praxisbezogenen Übersichtsartikeln zu den verschiedensten Themen der Rheumatologie und benachbarten Disziplinen beibehalten.

\section{Die erste Originalarbeit}

So wie die erste Ausgabe dieses Jahres die Premiere des neuen Layouts darstellte, freue ich mich, auch in dieser Ausgabe von rheuma plus sozusagen eine Uraufführung ankündigen zu können. Wir sind stolz darauf, die erste eingereichte und nach Review angenommene Originalarbeit in der Geschichte dieses Magazins publizieren zu können. In der Rubrik „Aus der Praxis“ präsentieren Oskar Psenak und Koll. von der Paracelsus Medizinischen Privatuniversität in Salzburg eine junge Dame mit SLE und Schwangerschaft unter immunsuppressiver Therapie. Das Thema Schwangerschaft gewinnt für uns Rheumatologen zunehmend an Bedeutung; vor allem, weil wir dank der modernen Therapiemöglichkeiten Gott sei Dank immer mehr in der Lage sind, die Patientinnen so zu behandeln, dass an eine Schwangerschaft zu denken ist.

Ziel unserer therapeutischen Bemühungen hat es immer zu sein, den Betroffenen ein möglicht normales Leben zu gewährleisten. In diesem Zusammenhang laden wir herzlich dazu ein, uns Beiträge $\mathrm{zu}$ allen rheumatologischen Themen, die Sie für relevant halten, zu schicken. Selbstverständlich kann nicht für jeden Beitrag eine Garantie auf Publikation abgegeben werden, aber auf einen fairen Review mit entsprechendem Feedback können Sie vertrauen.

Schmerzen sind es, welche die Patienten zum Rheumatologen führen, Schmerzen sind es, von denen eigentlich jeder Mensch befreit werden möchte, stellen sie doch einen der wesentlichsten Faktoren dar, welche die Lebensqualität beeinträchtigen. Dass Schmerzbehandlung eine komplexe Aufgabe darstellt, die einen multimodalen Ansatz verlangt, ist zwar heutzutage allgemein als Consensus anerkannt, dennoch sollte dieser auch immer wieder hinterfragt und kritisch-konstruktiv beleuchtet werden. Andrea Österbauer aus Hochzirl tut dies in dieser Ausgabe und bringt damit hoffentlich viel an Diskussionsstoff ein.

\section{Aktuelles vom EULAR-Kongress}

Einen weiteren Schwerpunkt dieser Ausgabe stellt wie immer der EULAR-Kongress dar. Traditionellerweise präsentiert die zweite Ausgabe von rheuma plus im Jahr österreichische Beiträge zum größten rheumatologischen Kongress auf der Welt, der dieses Jahr in Madrid veranstaltet wird.

Die Rubrik „BioReg berichtet“ wird sich diesmal vowiegend mit Patienten mit Rheumatoider Arthritis befassen: Welche Komorbiditäten bestehen, wie schaut es mit der TB-Prophylaxe aus, wie häufig werden Veränderungen an der Therapie durchgeführt, wie steht es um die Arbeitsfähigkeit? Dieses Register, obwohl nicht einmütig unterstützt, kann bereits getrost als Erfolgsgeschichte bezeichnet werden. Zum Zeitpunkt des Abfassens dieses Editorials sind bereits mehr als 1.300 Patienten mit entzündlich rheumatischen Erkrankungen und Biologika-Therapie systematisch dokumentiert.
Zweifellos heißt verantwortungsbewusste Medizin zu betreiben, auch immer wieder die bestehende Situation zu analysieren und hinterfragen. Das Ziel von rheuma plus ist es immer, eine fruchtbringende und offene Diskussion in Gang zu bringen, aus der wir alle Nutzen ziehen können.

Zum Abschluss möchte ich der Hoffnung Ausdruck geben, dass Sie in dieser Ausgabe für sich Interessantes finden konnten, das Ihnen in der Praxis von Nutzen sein kann. Wie immer sage ich für Kritik, Hinweise, Zustimmung und für jeden anderen Beitrag auch schon im Voraus Danke.

Herzlichst,

Ihr

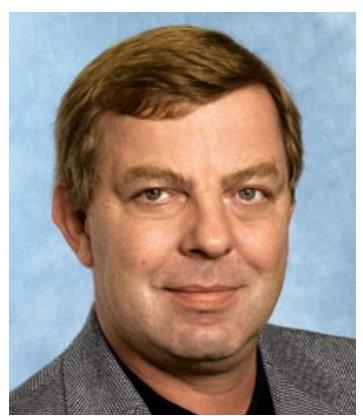

Burkhard Leeb 